\title{
MEN1-related hyperparathyroidism: response to cinacalcet and its relationship with the calcium-sensing receptor gene variant Arg990Gly
}

\author{
Marcello Filopanti ${ }^{1}$, Uberta Verga $^{1}$, Federica Ermetici $^{2}$, Luca Olgiati $^{1}$, Cristina Eller-Vainicher $^{1}$, Sabrina Corbetta ${ }^{3,4}$, \\ Luca Persani ${ }^{3,5}$, Paolo Beck-Peccoz ${ }^{1,3}$ and Anna Spada ${ }^{1,3}$ \\ ${ }^{1}$ Endocrinology and Diabetology Unit, Fondazione Istituto di Ricerca e Cura a Carattere Scientifico (IRCCS) Ca' Granda Ospedale Maggiore Policlinico, \\ Padiglione Granelli, via F. Sforza 35, 20122 Milan, Italy, ${ }^{2}$ Diabetology and Metabolic Disease Unit, IRCCS Policlinico San Donato, San Donato Milanese, \\ 20097 Milan, Italy, ${ }^{3}$ Department of Medical Sciences, University of Milan, 20122 Milan, Italy, ${ }^{4}$ Endocrinology and Diabetology Unit, IRCCS Policlinico \\ San Donato, San Donato Milanese, 20097 Milan, Italy and ${ }^{5}$ Division of Endocrine and Metabolic Diseases, and Laboratory of Endocrine and Metabolic \\ Research, IRCCS Istituto Auxologico Italiano, 20149 Milan, Italy
}

(Correspondence should be addressed to A Spada at Endocrinology and Diabetology Unit, Fondazione Istituto di Ricerca e Cura a Carattere Scientifico (IRCCS) Ca' Granda Ospedale Maggiore Policlinico; Email: anna.spada@unimi.it)

\begin{abstract}
Objective: Primary hyperparathyroidism (PHPT) is a challenging problem in type 1 multiple endocrine neoplasia (MEN1) due to the high postsurgery recurrence rate. The aim was to evaluate the efficacy of cinacalcet in MEN1 patients in comparison with patients with sporadic PHPT (sPHPT) and the effect of Arg990Gly calcium-sensing receptor (CASR) polymorphism on the response to treatment.

Design: This is a randomized, crossover, double-blind study carried out in the University Hospitals. Methods: Fifteen MEN1 patients with PHPT were randomized to two groups, one administered with $30 \mathrm{mg}$ daily cinacalcet, titrated until calcium normalization, and one with placebo. After 3 months, patients were reassessed and after washout switched to the other treatment. For comparison, 20 sPHPT patients with similar calcium levels were administered with cinacalcet for 3 months. Ionized and total calcium, phosphate, and parathyroid hormone (PTH) were evaluated. CASR Arg990Gly was genotyped on blood DNA by direct sequencing.

Results: Cinacalcet normalized calcium, increased phosphate, and reduced PTH levels in all patients. Cinacalcet dosage required to normalize calcium in MEN1 and SPHPT was not significantly different $(45 \pm 21$ vs $54 \pm 25 \mathrm{mg}$ /day). Few mild adverse events, not requiring drug withdrawal, were observed in both the groups. No association between Arg990Gly CASR polymorphism and response to cinacalcet was found.

Conclusions: This short-term prospective study demonstrated that the efficacy profile of cinacalcet in patients with MEN1-related PHPT and in those with SPHPT was similar and was not influenced by the 990 CASR variant. Although long-term safety and efficacy data are required, cinacalcet might be considered a treatment option in MEN1 patients who have contraindications to surgery or persistent PHPT after surgery.
\end{abstract}

European Journal of Endocrinology 167 157-164

\section{Introduction}

Multiple endocrine neoplasia type 1 (MEN1, OMIM $+131100)$ is an autosomal dominant disorder characterized by the combined occurrence of tumors of parathyroids, anterior pituitary, and pancreatic islet cells, due to inactivating mutations of MEN1 gene. Primary hyperparathyroidism (PHPT) is present in $100 \%$ of MEN1 patients at age 50 years, and renal and bone complications are more frequent in these patients than in patients with sporadic PHPT (sPHPT) $(1,2)$. Treatment options for PHPT are parathyroidectomy for patients meeting current criteria according to the National Institutes of Health's Consensus (2001) (1) and regular monitoring of biochemical markers and disease complications. Due to the frequent occurrence of multiple gland hyperplasia and ectopic locations, the success rate of surgery in MEN1 patients is definitely lower than that of patients with SPHPT, with a recurrence rate of about $50 \%$, even in the hands of experienced parathyroid surgeons $(2,3,4)$.

Cinacalcet is an allosteric modulator of the calciumsensing receptor (CASR) that sensitizes the receptor to the extracellular calcium. Cinacalcet is effective in reducing or normalizing serum calcium levels in patients with hyperparathyroidism of different origins $(5,6,7,8,9)$. According to the European Medicines Agency (EMA) prescription labeling, cinacalcet is 
indicated for the treatment of patients with secondary HPT due to chronic kidney disease, patients with PHPT due to parathyroid carcinoma, and patients with PHPT meeting the criteria for parathyroidectomy who have contraindications to surgery or persistent PHPT after surgery. Although MEN1 patients are good candidates for a medical treatment, the effectiveness of cinacalcet has been so far reported in 11 patients described in three different studies $(10,11,12)$.

In recent years, previous studies investigated the possible association between polymorphic variants of the CASR gene and clinical and biochemical characteristics of PHPT patients $(13,14)$. In a cohort of sPHPT patients previously reported, patients homozygous or heterozygous for the Arg990Gly polymorphism had a phenotype consistent with increased sensitivity of the variant 990Gly to extracellular calcium (15). At present, only one small cohort of patients with secondary HPT due to chronic kidney disease has been screened for possible association between CASR polymorphisms and different responsiveness to cinacalcet (16). In this study, patients bearing the 990Gly allele were more sensitive to cinacalcet compared with homozygotes for Arg at that location (16). This observation is consistent with in vitro functional data, showing increased sensitivity of HEK cells stably transfected with CASR 990Gly allele to the calcimimetic R-568 (17) and an increased antilipolytic effect of cinacalcet in primary cultures of normal adipocytes having 990Gly allele (18).

This study is a randomized, crossover, double-blind medical approach to PHPT in patients with MEN1, in analogy with that observed in patients with sPHPT. Moreover, the study will explore the possibility to identify patients with different responsiveness to the drug by a pharmacogenetic approach based on the polymorphic Arg990Gly variant of CASR gene.

\section{Patients and methods}

\section{Patients}

The study included 15 patients (eight females and seven males; age at presentation $42.3 \pm 12.2$ years) with genetically confirmed MEN1 affected by PHPT enrolled between June and December 2010 (Table 1) and 20 consecutive unrelated patients ( 16 females and four males, age $61 \pm 12.4$ years, $P<0.001$, vs MEN1 patients) with sPHPT matched with MEN1-related PHPT patients for serum calcium levels. Diagnosis of PHPT was made on the basis of high ionized calcium levels in the presence of elevated or inappropriately normal serum parathyroid hormone (PTH) levels in $1.44 \pm 0.5$ and $1.43 \pm 0.6 \mathrm{mmol} / \mathrm{l}, \quad P=\mathrm{NS}, \quad$ n.v. 1.13-1.32; total serum calcium $11.5 \pm 0.2$ and study aimed to test whether cinacalcet may represent a MEN1 and sPHPT patients (plasma ionized calcium

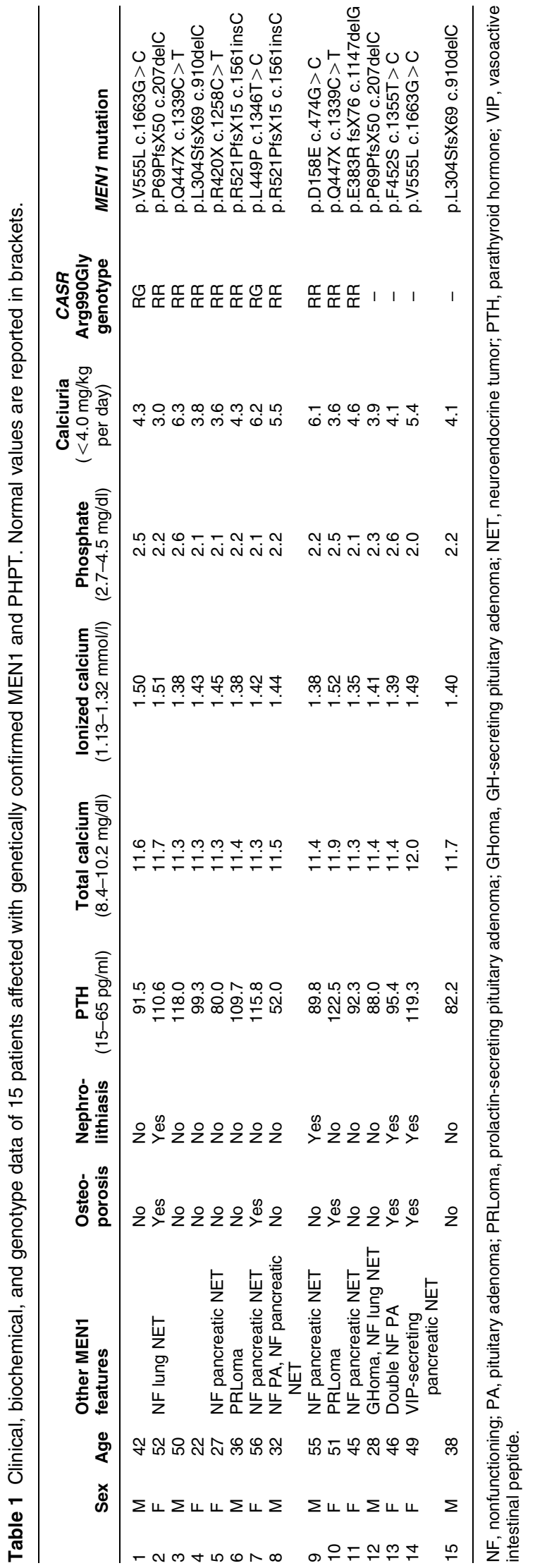

www.eje-online.org 
$11.7 \pm 0.5 \mathrm{mg} / \mathrm{dl}, P=\mathrm{NS}$, n.v. 8.4-10.2; serum $\mathrm{PTH}$ $97.8 \pm 18.9$ and $170.1 \pm 126.8 \mathrm{pg} / \mathrm{ml}, P=0.005$, n.v. $10-65 \mathrm{pg} / \mathrm{ml}$; calciuria $4.6 \pm 1.1$ and $4.8 \pm 1.5, P=\mathrm{NS}$, Table 1) and surgically confirmed in seven patients. Moreover, in all patients, blood chromogranin-A was measured $(41.2 \pm 19.5 \mathrm{U} / \mathrm{l}$, n.v. <35), whereas gastrin was evaluated in five patients $(66.8 \pm 25.0 \mathrm{pg} / \mathrm{ml}$, n.v. <108).

Systolic and diastolic blood pressures (SBP and DBP) were measured according to the World Health Organization (WHO) International Society of Hypertension Guidelines. Arterial hypertension (DBP > 85 and SBP $>135 \mathrm{mmHg}$ ) was present in $26.7 \%$ of MEN1 and $50 \%$ of sPHPT patients $(P=N S)$. Patients with systolic hypertension were treated with angiotensin-converting enzyme inhibitors, angiotensin II receptor antagonists, or $\beta$-adrenergic receptor antagonists with no diuretics in monotherapy or in association. Nephrolithiasis, defined as a history of renal colic with stone expulsions and/or imaging identification or asymptomatic ultrasound imaging of kidney stones, was identified in $40 \%$ of MEN1 and $50 \%$ of sPHPT patients $(P=N S)$. Osteoporosis, defined as a $T$-score less than -2.5 at least in one of the sites evaluated with dual-energy $\mathrm{X}$-ray absorptiometry at femur and lumbar spine levels, was present in $26.7 \%$ of MEN1 and $70 \%$ of sPHPT patients $(P<0.04)$.

All patients met the EMA criteria for cinacalcet treatment as they had persistent PHPT after surgery or contraindications to surgery, which would be indicated on the basis of the serum calcium level that was $\geq 1 \mathrm{mg}$ above the upper normal limit in all patients (as defined by relevant treatment guidelines). In particular, eight patients had PHPT relapse after one or more surgical operations, two patients had contraindications, and five patients refused or postponed surgery for personal reasons.

Written informed consent was obtained from all patients and the study was authorized by the local ethics committees and was registered with the EudraCT (European Clinical Trials) Database as 2008-005055-23.

\section{Study design}

The study had the assessment of the efficacy on calcium-phosphorus metabolism and safety profile of cinacalcet as primary clinical objective and the evaluation of the possible role of CASR 990Gly polymorphism on cinacalcet response in MEN1 patients as secondary objective. MEN1 patients were enrolled in a randomized, crossover, double-blind with $\mathrm{AB} / \mathrm{BA}$ design study. After baseline assessment of clinical and biochemical parameters, patients with MEN1 were randomly divided into two groups $(\mathrm{A} / \mathrm{B})$ and treated with cinacalcet and placebo respectively. Cinacalcet treatment was started with $30 \mathrm{mg}$ daily dosage and subsequently titrated according to the following algorithm: i) serum and ionized calcium was assessed after 1 week; ii) if not normalized, a dose of $30 \mathrm{mg}$ was added, returning to point 1 ; and iii) if normalized, cinacalcet dosage was kept constant and exposition phase started.

After titration, treatment was maintained for 3 months and then patients were reassessed for clinical and biochemical parameters. After 1 month of washout, treatments were switched (B/A) and patients underwent a second exposure phase and were finally reassessed. Titration dosage and clinical examination were carried out by different physicians in order to preserve doubleblind protocol. As regards blinding procedure and placebo controlling, cinacalcet and placebo tablets were wrapped in anonymous capsules and confections were labeled with alphanumeric codes unknown to patients and personnel dedicated to clinical examination. Before enrollment, all patients were treated with orally administered cholecalciferol 300000 units every 4-6 months and the last administration was between 1 and 2 months before initiation of the study. During the study phases, cholecalciferol replacement was suspended. None of the patients received bisphosphonates within 6 months.

For comparison, the efficacy of 3 months of cinacalcet treatment was evaluated in a cohort of 20 consecutive sPHPT patients, selected on the basis of pretreatment levels of serum calcium similar to those found in MEN1 patients. All patients were treated with orally administered cholecalciferol 300000 units every 4-6 months and the last administration was between 1 and 2 months before the initiation of study. None of these patients took cholecalciferol during the first 3 months after cinacalcet starting. Thirteen patients (65\%) regularly took bisphosphonates for osteoporosis for 9-48 months prior to assessment. After basal evaluation, all sPHPT patients were administered with cinacalcet $30 \mathrm{mg} /$ day, and a dose of $30 \mathrm{mg}$ was added until serum calcium normalization. Reassessment was carried out after 3 months, without treatment interruption.

During the study, both MEN1-related and sporadic hyperparathyroidism patients were on free diet.

\section{Assays}

Blood samples for measurement of total and ionized calcium, phosphorus, PTH, and biochemistry were collected at baseline and at each follow-up visit after an overnight fasting and before the morning dose of cinacalcet. Twenty-four-hour urine samples were collected for calcium-phosphorous metabolism evaluation. Serum intact PTH was measured by a chemiluminescent method (Nichols Institute Diagnostics, San Juan Capistrano, CA, USA), with intra- and interassay coefficients of variation of $<4.5$ and $<10.0 \%$ respectively. All other analytes were determined using standard methods. Calcium adjusted for albumin was calculated by the formula: total calcium $+0.8 \times(4.4-$ albumin $)$. 


\section{DNA extraction and genotyping}

All patients included in the study were genotyped to test the possible association between the polymorphic Arg990Gly variant (rs1042636, c.2977A>G, and p.Arg990Gly) of CASR and responsiveness to cinacalcet treatment, as previously reported (15). Briefly, blood DNA was extracted and PCRs were carried out in $50 \mu \mathrm{l}$ of reaction mix with $500 \mathrm{ng}$ of patient DNA, $25 \mathrm{pmol}$ of each primer, and $2.5 \mathrm{U}$ of AmpliTaq DNA polymerase (Applied Biosystems, Life Technologies Corp., Carlsbad, CA, USA). The PCRs were denaturated for $3 \mathrm{~min}$ at $95^{\circ} \mathrm{C}$ and followed by 35 cycles at $95^{\circ} \mathrm{C}$ for $1 \mathrm{~min}$, $54{ }^{\circ} \mathrm{C}$ for $1 \mathrm{~min}$, and $72{ }^{\circ} \mathrm{C}$ for $1 \mathrm{~min}$. A final cycle at $72{ }^{\circ} \mathrm{C}$ for $10 \mathrm{~min}$ was carried out. The primer pair was 5'-GCAAGCAGAAGGTCATCTTT-3' and 5'-GTCCTTGCAGACCTGTTTC-3'. PCR products were sequenced by ABI-PRISM sequencer (Applied Biosystems, Life Technologies Corp.).

\section{Statistical analysis}

As the study design was a crossover AB/BA type, data pre- and posttreatment were analyzed with paired Student's t-test (Gaussian parameters) or by McNemar's test (categorical parameters). Comparisons with sPHPT patients were performed by unpaired Student's $t$-test for continuous variables and $\chi^{2}$ test for categorical variables. PTH and calcium vs cinacalcet dosage bivariate correlation was evaluated by Spearman's rank correlation coefficient. $P<0.05$ was considered statistically significant. Calculations were made by SPSS 18.0 software (IBM, Inc., Armonk, NY, USA).

\section{Results}

After randomization of MEN1 patients, seven patients received cinacalcet (group A) and eight patients were administered with placebo (group B). After titration, the mean cinacalcet dosage was $40 \pm 16 \mathrm{mg} /$ day. In this group, minimal cinacalcet dosage able to normalize calcium levels was reached in 1-2 weeks. One patient of group A experienced transient nausea for about 1 week (cinacalcet dosage: $30 \mathrm{mg} /$ day), without necessity of treatment withdrawal. No patients of group A had hypocalcemia. During the first maintenance phase ( 3 months), one patient of group $\mathrm{A}$ and two of group B exited from the study due to personal reasons.

Subsequently, patients were reassessed for clinical and biochemical parameters and, after 1-month washout, treatments were switched between the two groups and the second maintenance phase started. After titration, the mean cinacalcet dosage was $48 \pm 27 \mathrm{mg} /$ day and the minimal effective dosage was reached in 1-3 weeks. One of the group B patients experienced transient nausea for few days, without treatment interruption or modification (cinacalcet dosage: $60 \mathrm{mg} /$ day). No patients had hypocalcemia. At the first visit of the second maintenance phase, one patient of the placebo group exited from the study for personal reasons. After 3 months, patients were reassessed and the study ended.

Paired comparisons between cinacalcet and placebo exposures of MEN1 patients who fully completed the study are presented in Table 2. In particular, during cinacalcet phase, plasma ionized and serum albumincorrected calcium were normalized in all patients and were accompanied by a significant increase in serum phosphate (Table 2 and Fig. 1). As far as PTH levels were considered, a statistically significant reduction of PTH levels occurred in all patients (median - 26.3\%, interquartile range (IQR) $19.2-43.6 \%, P=0.002$ ), with normalization in five patients $(45.4 \%)$ (Table 2 and Fig. 1). No significant change in 24-h urinary calcium $(4.8 \pm 1.2 \mathrm{mg} / \mathrm{kg}$ per day, $P=\mathrm{NS})$, chromogranin A $(47.0 \pm 20.8, P=\mathrm{NS})$, and gastrin $(81.3 \pm 18.8 \mathrm{pg} / \mathrm{ml}$,

Table 2 Placebo and cinacalcet effects on PTH, calcium/phosphorous metabolism, and symptoms in MEN1-related and sporadic PHPT patients.

\begin{tabular}{|c|c|c|c|c|c|c|}
\hline & \multicolumn{3}{|c|}{ MEN1-related PHPT $(n=11)$} & \multicolumn{3}{|c|}{ Sporadic PHPT $(n=20)$} \\
\hline & Placebo & Cinacalcet & $P$ & Pretreatment & Cinacalcet & $P$ \\
\hline PTH (pg/ml) & $98.9 \pm 21.5$ & $68.5 \pm 22.3$ & 0.006 & $181.3 \pm 115.5$ & $120.7 \pm 38.8$ & 0.032 \\
\hline Corrected total serum calcium (mg/dl) & $11.5 \pm 0.2$ & $9.5 \pm 0.4$ & $<0.001$ & $11.7 \pm 0.5$ & $9.5 \pm 0.4$ & $<0.001$ \\
\hline Ionized calcium (mEq/l) & $1.44 \pm 0.5$ & $1.20 \pm 0.6$ & $<0.001$ & $1.43 \pm 0.6$ & $1.21 \pm 0.7$ & $<0.001$ \\
\hline Serum phosphate (mg/dl) & $2.3 \pm 0.2$ & $3.1 \pm 0.2$ & $<0.001$ & $2.5 \pm 0.3$ & $3.1 \pm 0.2$ & $<0.001$ \\
\hline Alkaline phosphatases (U/l) & $80 \pm 5$ & $72 \pm 12$ & 0.086 & $97 \pm 58$ & $93 \pm 47$ & 0.813 \\
\hline Serum creatinine $(\mathrm{mg} / \mathrm{dl})$ & $0.7 \pm 0.2$ & $0.9 \pm 0.2$ & 0.283 & $0.9 \pm 0.1$ & $0.8 \pm 0.1$ & 0.390 \\
\hline Creatinine clearance $(\mathrm{mg} / \mathrm{min})$ & $88.3 \pm 16.2$ & $87.7 \pm 22.3$ & 0.969 & $92.3 \pm 24.6$ & $77.4 \pm 40.3$ & 0.588 \\
\hline Daily urinary calcium (mg/day) & $323 \pm 124$ & $378 \pm 21$ & 0.585 & $295 \pm 109$ & $256 \pm 142$ & 0.658 \\
\hline Gastrointestinal symptoms $(\%, n)$ & $36 . \overline{4}(4)$ & $27 . \overline{3}(3)$ & 1.000 & $35 . \overline{0}(7)$ & $25.0(5)$ & 0.480 \\
\hline Neuromuscular symptoms $(\%, n)$ & $27.3(3)$ & $27.3(3)$ & 0.479 & $40.0(8)$ & $40.0(8)$ & 0.497 \\
\hline $\begin{array}{l}\text { Cinacalcet dosage (mg/day) } \\
\text { Median (IQR) }\end{array}$ & - & $\begin{array}{c}45 \pm 21 \\
30(30-60)\end{array}$ & - & - & $\begin{array}{c}54 \pm 25 \\
60(30-60)\end{array}$ & - \\
\hline
\end{tabular}

$I Q R$, interquartile range.

Statistically significant results are highlighted in bold. 
$P=$ NS) was found. Similarly, no significant variations in gastrointestinal and neuromuscular symptoms, blood pressure, and antihypertensive drug requirement between cinacalcet and placebo groups were observed (Table 2 and data not shown). As far as quality of life was concerned, similar scale scores were recorded in cinacalcet and placebo treatments (data not shown).

In the sPHPT group, cinacalcet normalized ionized and total calcium, increased serum phosphate, and did not change urinary calcium excretion in all patients. PTH normalization was obtained in only one patient (5.0\%), despite a percentage of PTH reduction (median - 20.6\%, IQR 14.9-33.3\%, $P=0.007$ ) (Table 2 and Fig. 1) that was similar to that recorded in MEN1 patients. In particular, no difference in PTH and calcium during cinacalcet treatment was observed between

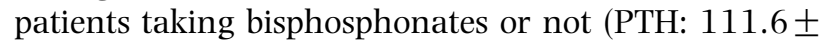
32.7 vs $137.6 \pm 46.1 \mathrm{pg} / \mathrm{ml}, P=\mathrm{NS}$, calcium: $9.6 \pm 0.4$ vs $9.4 \pm 0.4 \mathrm{mg} / \mathrm{dl}, P=\mathrm{NS})$. As regards adverse effects, four patients experienced nausea (one patient taking $30 \mathrm{mg} / \mathrm{day}$, two $60 \mathrm{mg} / \mathrm{day}$, and one $120 \mathrm{mg} / \mathrm{day})$, which lasted for a few days in three of them, without cinacalcet discontinuation. In one patient (dosage: $120 \mathrm{mg} /$ day), cinacalcet was maintained with the addition of ranitidine hydrochloride $150 \mathrm{mg} /$ day for 5 weeks, with symptom improvement.

Mean cinacalcet dosage required to control calcium levels in SPHPT patients had a tendency to be higher than that administered to MEN1 patients $(54 \pm 25$ vs $45 \pm 21 \mathrm{mg} /$ day, $P=0.314)$. In particular, in the maintenance phase, hypercalcemia was controlled by
$30 \mathrm{mg}$ daily in seven of 11 MEN1 and eight of 20 sPHPT patients $(P=\mathrm{NS})$. No significant correlations were found between cinacalcet dosage and both PTH and serum calcium reduction in MEN1, sPHPT, and MEN1 plus sPHPT groups (Fig. 2). No significant variations in SBP and DBP and the antihypertensive drug requirement between cinacalcet and placebo treatments were observed (Table 2 and data not shown).

Both MEN1 and sPHPT patients were genotyped for Arg990Gly polymorphism. Among MEN1 subjects who completed the study, nine patients $(81.8 \%)$ were Arg990 homozygotes and two were heterozygotes, while in sPHPT group, 17 patients (85.0\%) were Arg990 homozygotes and three were heterozygotes. No striking differences in PTH, serum calcium, phosphorous, nephrolithiasis, and osteoporosis were observed either at baseline or after treatment between 990Gly carriers and noncarriers, both in MEN1 and sPHPT groups considered separately or together. Moreover, no significant difference was found in cinacalcet dosage required for calcium normalization and PTH reduction between patients carrying the Gly allele or not (Fig. 2).

At the end of the study, four out of six patients (two females), who took cinacalcet during the second maintenance phases, agreed to continue the treatment for an unblind observational period of 12 months and were regularly assessed likewise the previously described maintenance phase. No variation in cinacalcet dosage was needed as calcium and PTH remained stable and no adverse effects appeared. Lumbar spine and
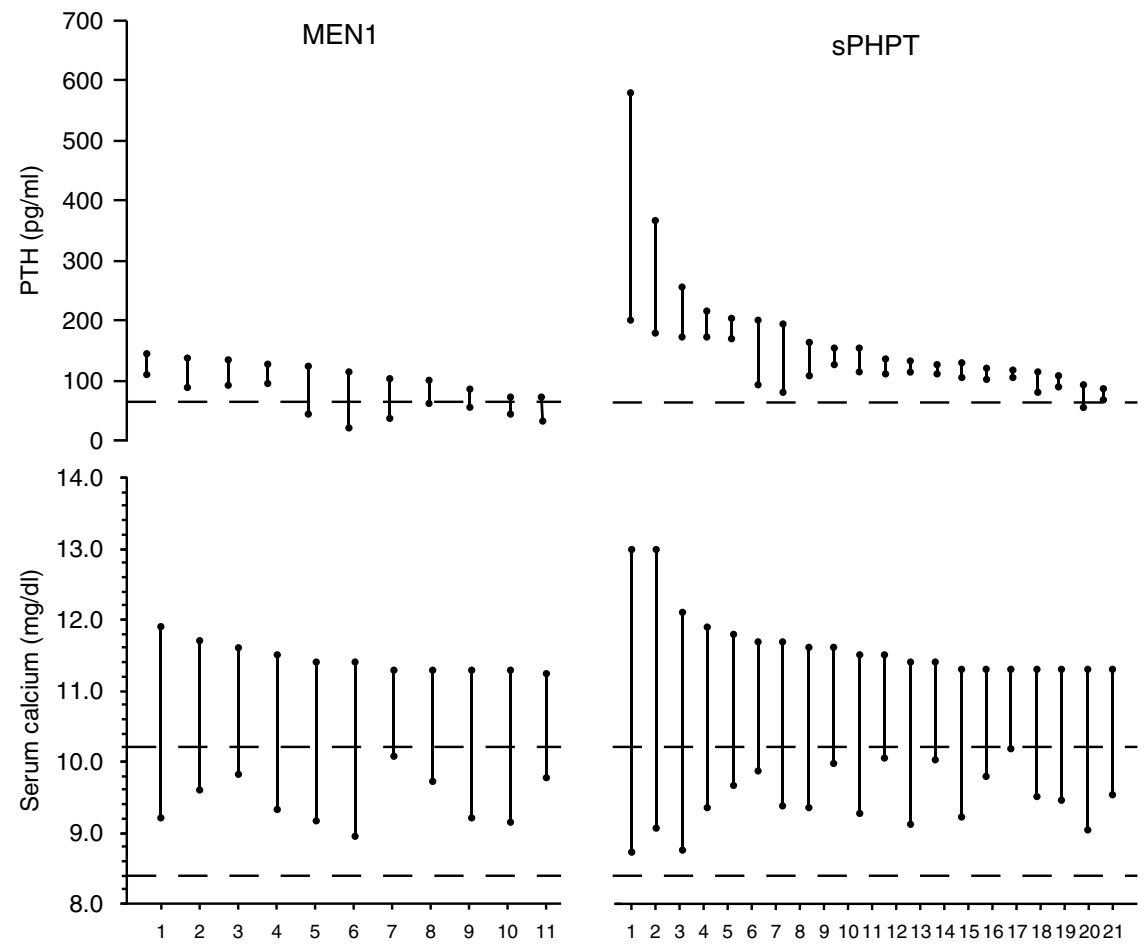

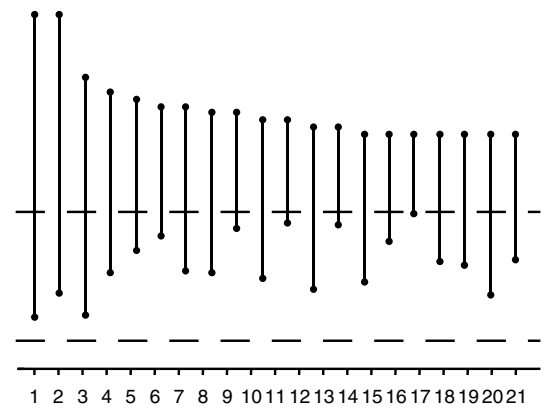

Figure $1 \mathrm{PTH}$ and albumin-corrected serum calcium level reductions after 3 months of cinacalcet treatment in 11 patients with MEN1 and 20 patients with sPHPT. 


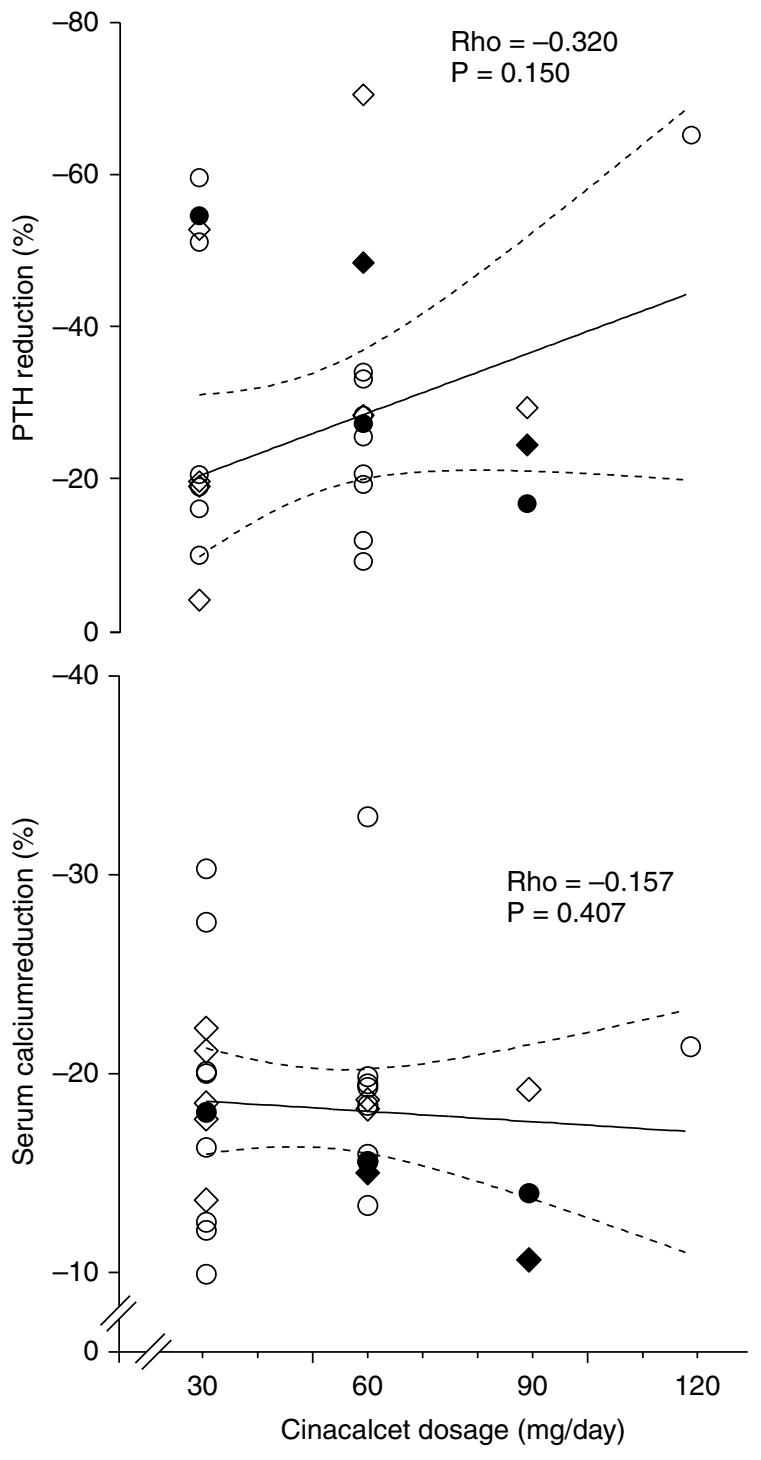

Figure 2 Correlation between cinacalcet dosage and PTH and albumin-corrected serum calcium percent reduction after 3 months of treatment in 31 patients affected with both sporadic and MEN1-related primary hyperparathyroidism. Open circles, sPHPT patients; Open squares, MEN1-related hyperparathyroidism patients. Closed circles, sPHPT patients 990Gly cariers; closed squares, MEN1-related hyperparathyroidism patients 990Gly carriers. $\rho$, Spearman's correlation coefficient.

femur bone densitometry, which was repeated after 12 months of continuous treatment, did not show any significant variation in bone mass density.

\section{Discussion}

This short-term prospective study confirms that cinacalcet is effective in controlling hypercalcemia in patients with PHPT and extends this observation to patients with MEN1-related PHPT. It is well established that cinacalcet, a drug able to sensitize CASR to extracellular calcium, is a treatment option for patients with PHPT for whom parathyroidectomy would be indicated according to the National Institutes of Health's Consensus, but in whom surgery is refused or contraindicated. Due to the frequent occurrence of multiple gland hyperplasia and ectopic locations, the success rate of parathyroid surgery in MEN1 patients is definitely lower than that of SPHPT, with a recurrence rate of about $50 \%$ of patients in the hands of experienced parathyroid surgeons $(2,3,4)$. Although MEN1-related PHPT patients are likely the best candidates for a medical treatment, the effectiveness of cinacalcet in MEN1 has been poorly investigated, probably due to low frequency of the disorder that is estimated in two to ten cases per 100000 in the general population. Indeed, three different studies described the efficacy of cinacalcet in a total of 11 patients $(10,11,12)$.

In this study, 15 MEN1 patients were enrolled in a randomized, crossover, double-blind with $\mathrm{AB} / \mathrm{BA}$ design study, and the efficacy and safety profile of cinacalcet were compared with that found in a group of $20 \mathrm{sPHPT}$ patients, who were matched for calcium levels and not for age and sex due to the well-known difference between MEN1-related and SPHPT in these parameters. The short-term administration ( 3 months) of cinacalcet induced the normalization of ionized and total calcium that was accompanied by a significant increase in serum phosphate, no change in urinary calcium excretion, chromogranin-A, and gastrin in all MEN1 patients, while PTH levels were reduced in all patients, reaching the normal range in about half the number of patients. Admittedly, parameters associated with hypercalcemia, such as gastrointestinal and neuromuscular symptoms, blood pressure, and quality of life, did not improve during cinacalcet, possibly due to the short treatment duration. Normalization of calcium and PTH levels was confirmed in a small subgroup of four patients who agreed to continue cinacalcet for an additional unblind observational phase of 12 months. As previously reported in sPHPT patients (19), no significant variations of bone mass density were observed in these patients.

In the sPHPT group, while calcium normalization was obtained in all patients, PTH reduction within the normal range was observed in one only patient. These data are consistent with the previous observation of a more impressive reduction of PTH levels observed in individual MEN1 patients in comparison with that obtained in sPHPT $(11,12)$. The underlying pathology may be of importance in determining the different response to cinacalcet, as the expression level of CASR, which is the drug target, might be different in MEN1associated chief cell hyperplasia in comparison with sporadic adenoma. However, as in this study the percent PTH decrease reached during cinacalcet treatment was similar in MEN1 and SPHPT patients, the difference in the rate of PTH normalization between the two groups 
was probably related to pretreatment PTH levels that were significantly higher in SPHPT in comparison with MEN1-related PHPT. In fact, as reported in a previous multicenter study on a large series of sporadic and MEN1-related PHPT, in addition to young age, the presence of normal, but inappropriate, PTH levels is a hallmark of MEN1 (19). Despite the rare occurrence of PTH normalization in SPHPT patients, the percent PTH decline observed in this cohort of patients $(33 \%)$ was greater than that previously reported $(8,9,19)$, probably related to a less severe disorder in our series. Indeed, in order to comparatively assess the efficacy of cinacalcet on calcium normalization in MEN1 patients, sPHPT patients were selected on the basis of pretreatment levels of serum calcium similar to those found in MEN1 patients. Moreover, the possible contribution of vitamin D administration on PTH reduction in MEN1 and sPHPT patients was ruled out as deficient patients were appropriately supplemented every 4-6 months before the enrollment.

A low rate of adverse events, usually mild and transient nausea that did not require treatment withdrawal, was observed in both MEN1 and sPHPT patients. These results were probably due to the low dosage of cinacalcet $(45 \pm 21 \mathrm{mg} /$ day in MEN1 and $54 \pm 25 \mathrm{mg} /$ day in sPHPT) effective in restoring normocalcemia, as greater frequency and severity of side effects have been reported in patients requiring high dose (up to $60 \mathrm{mg}$ twice daily) to control hypercalcemia $(8,20,21)$. As far as cinacalcet dosage was concerned, this study did not confirm the higher sensitivity to cinacalcet of MEN1 patients, which has been suggested on the basis of clinical and biochemical data of case reports $(11,12)$. In fact, the percentage of patients requiring $30 \mathrm{mg}$ cinacalcet daily to restore normocalcemia did not significantly differ between the MEN1 and sPHPT groups.

This study investigated the possible association of the Arg990Gly variant of the CASR gene to the clinical and biochemical response to cinacalcet. Several polymorphisms within the human CASR gene have been described in healthy populations and patients with disorders in calcium metabolism. In particular, the contribution of Arg990Gly to the clinical course and severity of PHPT has been extensively investigated $(13,14)$. Although data are not conclusive, there is evidence to suggest that patients with the 990Gly allele had a phenotype consistent with increased sensitivity of the receptor to extracellular calcium $(15,17,22)$. Indeed, Arg990Gly is a nonconservative polymorphism located in the CASR carboxyl-terminal tail, a domain that impacts on several receptor properties, such as cell surface expression, intracellular signaling activation, and desensitization (23). Moreover, the gain of function of this variant has been confirmed by in vitro experiments showing the increased sensitivity of HEK cells stably transfected with CASR 990Gly allele to the calcimimetic R-568 (17).
At present, only a cohort of seven patients with secondary HPT due to chronic kidney disease has been screened for possible association between CASR polymorphisms and responsiveness to cinacalcet (16). In that study, patients bearing the 990Gly allele showed a more marked inhibition of PTH levels in response to cinacalcet compared with those homozygous for Arg at that location (16).

The data reported here did not support an influence of the variant receptor on the response to cinacalcet $(16,24)$, although the small set of patients investigated by this study as well as by the previous one did not allow definitive conclusions.

In conclusion, this short-term study demonstrated that the efficacy and safety profile of cinacalcet in patients with MEN1-related PHPT and in patients with SPHPT was similar and that the response to cinacalcet treatment was not associated with 990 CASR variant. Due to the low occurrence of successful parathyroid surgery and the high recurrence rate in MEN1 patients, cinacalcet might be considered a second choice treatment option for calcium normalization in these patients. However, it is worth noting that normalization of calcium and $\mathrm{PTH}$ levels was not associated with improvements in calciuria, bone density, symptoms, or quality of life, possibly due to the short-term treatment. Moreover, the cost of this option should be taken into account, also considering the rather young age of many MEN1-related PHPT patients. Finally, the lack of safety data of long-term cinacalcet treatment and its poor, if any, beneficial effect on bone mass are important limitations that require further clinical investigations. Similarly, global, double-blind, randomized, placebo-controlled trials are very eagerly awaited for the evaluation of whether normalization of calcium and PTH by long-term cinacalcet treatment reduces the increased cardiovascular morbidity and mortality in PHPT patients.

\section{Declaration of interest}

The authors declare that there is no conflict of interest that could be perceived as prejudicing the impartiality of the research reported.

\section{Funding}

This study was supported by Italian Drugs Agency AIFA 'Bando Ricerca Indipendente sui Farmaci 2007' FARM754KTS grant.

\section{Acknowledgements}

The authors thank Mrs Renata Ciusani for placebo preparation.

\section{References}

1 Brandi ML, Gagel RF, Angeli A, Bilezikian JP, Beck-Peccoz P, Bordi C, Conte-Devolx B, Falchetti A, Gheri RG, Libroia A, Lips CJ, Lombardi G, Mannelli M, Pacini F, Ponder BA, Raue F, Skogseid B, Tamburrano G, Thakker RV, Thompson NW, Tomassetti P, Tonelli F, Wells SA Jr \& Marx SJ. Guidelines for diagnosis and 
therapy of MEN type 1 and type 2. Journal of Clinical Endocrinology and Metabolism 200186 5658-5671. (doi:10.1210/jc.86.12. 5658)

2 Tonelli F, Marcucci T, Fratini G, Tommasi MS, Falchetti A \& Brandi ML. Is total parathyroidectomy the treatment of choice for hyperparathyroidism in multiple endocrine neoplasia type 1? Annals of Surgery 2007246 1075-1082. (doi:10.1097/SLA. 0b013e31811f4467)

3 Hubbard JG, Sebag F, Maweja S \& Henry JF. Subtotal parathyroidectomy as an adequate treatment for primary hyperparathyroidism in multiple endocrine neoplasia type 1. Archives of Surgery 2006141 235-239. (doi:10.1001/archsurg.141.3.235)

4 Lambert LA, Shapiro SE, Lee JE, Perrier ND, Truonq M, Wallace MJ, Hoff AO, Gagel RF \& Evans DB. Surgical treatment of hyperparathyroidism in patients with multiple endocrine neoplasia type 1. Archives of Surgery 2005140 374-382. (doi:10.1001/archsurg. 140.4.374)

5 Wuthrich RP, Martin D \& Bilezikian JB. The role of calcimimetics in the treatment of hyperparathyroidism. European Journal of Clinical Investigation $2007 \quad 37$ 915-922. (doi:10.1111/j.1365-2362. 2007.01874.x)

6 Collins MT, Skarulis MC, Bilezikian JP, Silverberg SJ, Spiegel AM \& Marx SJ. Treatment of hypercalcemia secondary to parathyroid carcinoma with a novel calcimimetic agent. Journal of Clinical Endocrinology and Metabolism 199883 1083-1088. (doi:10. 1210/jc.83.4.1083)

7 Silverberg SJ, Rubin MR, Faiman C, Peacock M, Shoback DM \& Smallridge RC. Cinacalcet hydrochloride reduces the serum calcium concentration in inoperable parathyroid carcinoma. Journal of Clinical Endocrinology and Metabolism $2007 \mathbf{9 2}$ 3803-3808. (doi:10.1210/jc.2007-0585)

8 Peacock M, Bilezikian JP, Klassen PS, Guo MD, Turner SA \& Shoback D. Cinacalcet hydrochloride maintains long-term normocalcemia in patients with primary hyperparathyroidism. Journal of Clinical Endocrinology and Metabolism 200590 135-141. (doi:10.1210/jc.2004-0842)

9 Shoback DM, Bilezikian JP, Turner SA, McCary LC, Guo MD \& Peacock M. The calcimimetic cinacalcet normalizes serum calcium in subjects with primary hyperparathyroidism. Journal of Clinical Endocrinology and Metabolism 200388 5644-5649. (doi:10.1210/jc.2002-021597)

10 Falchetti A, Cilotti A, Vaggelli L, Masi L, Amedei A, Cioppi F, Tonelli F \& Brandi ML. A patient with MEN1-associated hyperparathyroidism, responsive to cinacalcet. Nature Clinical Practice. Endocrinology \& Metabolism 20084 351-357. (doi:10. 1038/ncpendmet0816)

11 Moyes VJ, Monson JP, Chew SL \& Akker SA. Clinical use of cinacalcet in MEN1 hyperparathyroidism. International Journal of Endocrinology 20102010 906163. (doi:10.1155/2010/906163)

12 Cetani F, Saponaro F, Banti C, Cianferotti L, Vignali E, Chiavistelli S, Viccica G, Pinchera A \& Marcocci C. Cinacalcet efficacy in patients with moderately severe primary hyperparathyroidism according to the European Medicine Agency prescription labeling. Journal of Endocrinological Investigation, 2011. (doi:10.3275/7970)

13 Yamauchi M, Sugimoto T, Yamaguchi T, Yano S, Kanzawa M, Kobayashi A \& Chihara K. Association of polymorphic alleles of the calcium-sensing receptor gene with the clinical severity of primary hyperparathyroidism. Clinical Endocrinology 200155 373-379. (doi:10.1046/j.1365-2265.2001.01318.x)

14 Cetani F, Borsari S, Vignali E, Pardi E, Picone A, Cianferotti L, Rossi G, Miccoli P, Pinchera A \& Marcocci C. Calcium-sensing receptor gene polymorphisms in primary hyperparathyroidism. Journal of Endocrinological Investigation 200225 614-619.
15 Corbetta S, Eller-Vainicher C, Filopanti M, Saeli P, Vezzoli G, Arcidiacono T, Loli P, Syren ML, Soldati L, Beck-Peccoz P \& Spada A. R990G polymorphism of the calcium-sensing receptor and renal calcium excretion in patients with primary hyperparathyroidism. European Journal of Endocrinology 2006155 687-692. (doi:10.1530/eje.1.02286)

16 Rothe HM, Shapiro WB, Sun WY \& Chou S-Y. Calcium-sensing receptor gene polymorphism R990G and its possible effect on response to cinacalcet HCl. Pharmacogenetics and Genomics 2005 15 29-34. (doi:10.1097/01213011-200501000-00005)

17 Terranegra A, Ferraretto A, Dogliotti E, Scarpellini M, Corbetta S, Barbieri AM, Spada A, Arcidiacono T, Rainone F, Aloia A, Cusi D, Vezzoli G \& Soldati L. Calcimimetic R-568 effects on activity of R990G polymorphism of calcium-sensing receptor. Journal of Molecular Endocrinology 201045 245-256. (doi:10.1677/JME10-0034)

18 Reyes M, Rothe HM, Mattar P, Shapiro WB \& Cifuentes M. Antilipolytic effect of calcimimetics depends on the allelic variant of calcium-sensing receptor gene polymorphism rs1042636 (Arg990Gly). European Journal of Human Genetics 201120 480-482. (doi:10.1038/ejhg.2011.221)

19 Peacock M, Bolognese MA, Borofsky M, Scumpia S, Sterling LR, Cheng S \& Shoback D. Cinacalcet treatment of primary hyperparathyroidism: biochemical and bone densitometric outcomes in a five-year study. Journal of Clinical Endocrinology and Metabolism 200994 4860-4867. (doi:10.1210/jc.2009-1472)

20 Eller-Vainicher C, Chiodini I, Battista C, Viti R, Mascia ML, Massironi S, Peracchi M, D’Agruma L, Minisola S, Corbetta S, Cole DE, Spada A \& Scillitani A. Sporadic and MEN1-related primary hyperparathyroidism: differences in clinical expression and severity. Journal of Bone and Mineral Research 200924 1404-1410. (doi:10.1359/jbmr.090304)

21 Marcocci C, Chanson P, Shoback D, Bilezikian J. Fernandez-Cruz L, Orgiazzi J, Henzen C, Cheng S, Ren Sterling L, Lu J \& Peacock M. Cinacalcet reduces serum calcium concentrations in patients with intractable primary hyperparathyroidism. Journal of Clinical Endocrinology and Metabolism 200994 2766-2772. (doi:10. $1210 /$ jc. 2008-2640)

22 Scillitani A, Guarnieri V, Battista C, De Geronimo S, Muscarella LA, Chiodini I, Cignarelli M, Minisola S, Bertoldo F, Francucci CM, Malavolta N, Piovesan A, Mascia ML, Muscarella S, Hendy GN, D'Agruma L \& Cole DE. Primary hyperparathyroidism and the presence of kidney stones are associated with different haplotypes of the calcium-sensing receptor. Journal of Clinical Endocrinology and Metabolism 200792 277-283. (doi:10.1210/ jc.2006-0857)

23 Hjalm G, MacLeod RH, Kifor O, Chattopadhyay N \& Brown EM. Filamin-A binds to the carboxyl-terminal tail of the calciumsensing receptor, an interaction that participates in CaR-mediated activation of mitogen-activated protein kinase. Journal of Biological Chemistry 2001276 34880-34887. (doi:10.1074/ jbc.M100784200)

24 Rothe HM, Liangos O, Biggar P, Petermann A \& Ketteler M. Cinacalcet treatment of primary hyperparathyroidism. International Journal of Endocrinology 20112011 Article ID 415719. (doi:10.1155/2011/415719)

Received 8 February 2012

Revised version received 2 May 2012

Accepted 10 May 2012 